\title{
A importância da pesquisa regulatória para um órgão regulador no setor nuclear
}

\author{
$\underline{\text { Sandra Regina Cabidolusso Lavalle Heilbron }}{ }^{1,2}$; Stella Regina Reis da Costa ${ }^{2}$ \\ sandra@cnen.gov.br
}

\section{Universidade Federal de Juiz de Fora, Faculdade de Engenharia - Campus da UFJF, 4a Plataforma do Setor de Tecnologia, Juiz de Fora, Brasil. \\ 2. Universidade Federal Fluminense - Escola de Engenharia, Rua Passo da Pátria, 156, Niterói, Brasil.}

Histórico do Artigo:

Recebido: 7 de março de 2017

Aceito: 14 de junho de 2017

Publicado: 18 de julho de 2017

Resumo: Um dos aspectos mais importantes a ser discutido em uma agência reguladora é a questão da necessidade da mesma priorizar a pesquisa regulatória. Este artigo apresenta a importância da pesquisa regulatória no âmbito das agências reguladoras internacionais, a importância dada pela Agência Internacional de Energia Atômica (AIEA) a este assunto, bem como alguns exemplos de pesquisa regulatória que vem sendo desenvolvidos atualmente na Comissão Nacional de Energia Nuclear (CNEN), pela área de segurança, onde fica demonstrado que uma agência reguladora nuclear não pode prescindir de fortalecer a área da pesquisa regulatória.

Palavras-chave: Pesquisa regulatória, Órgão regulatório, Setor nuclear, Órgão independente.

\section{The importance of regulatory research for a regulatory body in the nuclear sector}

\begin{abstract}
One of the most important aspects to be discussed in a regulatory agency is the issue of the need to prioritize regulatory research. This article presents the importance of regulatory research in international regulatory agencies, the importance given by the International Atomic Energy Agency (IAEA) to this issue, as well as some examples of regulatory research currently underway in the National Nuclear Energy Commission CNEN) by the area of security where it is demonstrated that a nuclear regulatory agency for the sector can not do without strengthening the area of regulatory research.

Keywords: Regulatory Research, Regulatory body, Nuclear sector, Independent body.
\end{abstract}




\section{La importancia de la investigación regulatória para una autoridad reguladora en el sector nuclear}

Resumen: Uno de los aspectos más importantes que se discutirán en una agencia reguladora es la cuestión de la necesidad de priorizar la misma investigación regulatoria. Este artículo presenta la importancia de la investigación regulatoria en los organismos reguladores internacionales, la importancia dada por la Agencia Internacional de Energía Atómica (OIEA) a este tema, así como algunos ejemplos de investigación regulatorio que se está desarrollando actualmente en la Comisión Nacional de Energía Nuclear ( CNEN) para la seguridad de la zona donde se demuestra que una agencia reguladora nuclear para el sector no puede prescindir de fortalecer el área de investigación de reglamentación.

Palabras clave: Investigación regulatória, Autoridad reguladora, Normativo, Industria nuclear, Autoridad independiente.

\section{INTRODUÇÃO}

A grande maioria dos regulamentos brasileiros sobre segurança nuclear e proteção radiológica, licenciamento de instalações nucleares, radiativas e mínero-industriais, gerência de rejeitos radioativos e controle de materiais radioativos, entre outros, toma por base regulamentos adotados internacionalmente. Esses regulamentos, os quais se fundamentam em modelos desenvolvidos para, por exemplo, (i) obter limites de isenção ou de exclusão dos processos de licenciamento nuclear e radiológico, (ii) definir testes para controle de qualidade, (iii) estabelecer margens de segurança, (iv) definir critérios para liberação de efluentes contendo poluentes líquidos e gasosos; podem, algumas vezes, ser aplicáveis em nosso país. No entanto, muitas hipóteses, condições de contorno e dados utilizados nesses modelos não refletem as condições geográficas, ambientais e socioeconômicas do Brasil.

A ausência de pesquisa regulatória, isto é, de uma análise científica de hipóteses, modelos e parâmetros aplicáveis ao Brasil, induz à adoção, "sem visão”, de regulamentos internacionais que podem vir a causar prejuízos financeiros e sociais para o país. Esse fato, por si só, ilustra a importância do investimento em pesquisa regulatória por parte de todo e qualquer país que seja soberano.

Outro grave aspecto relacionado à adoção de regulamentos estrangeiros, sem uma análise científica crítica, é o risco de que sejam impostas "barreiras não alfandegárias”, isto é, 
o estabelecimento de valores, limites e condicionantes falsamente relacionados a questões de segurança, quando, na verdade, o único objetivo é a garantia de reserva de mercado.

Importante ainda mencionar que para uma correta avaliação de segurança dos riscos associados a todas as instalações do setor nuclear, torna-se quase sempre necessário o desenvolvimento de modelos de avaliação de segurança próprios e, obviamente, independentes daqueles utilizados pelos operadores dessas instalações em suas próprias análises.

Ressalta-se que esses modelos são geralmente desenvolvidos em plataformas computacionais simbólicas, como do tipo "MATHEMATICA”, ou não simbólicas, como no caso do FORTRAN.

A aquisição de alguns códigos computacionais internacionais (na verdade, poucos são aqueles reconhecidos e consagrados pelas autoridades regulatórias mundiais) também tem sua importância, já que podem ser úteis para a verificação dos modelos desenvolvidos no país. Cabe observar, no entanto, que nem sempre estão disponíveis códigos adequados e aplicáveis às análises desejadas, já que a maioria deles utilizam dados ambientais, meteorológicos, propriedades de solo, biosfera e geosfera, limites, fatores de dose, entre outros, não necessariamente aplicáveis ao Brasil.

\section{MATERIAL E MÉTODOS}

De maneira a demonstrar a importância da pesquisa regulatória no âmbito de uma agência reguladora, foi feita uma análise sobre a importância da pesquisa regulatória atualmente para a área de segurança da CNEN e para as principais agências nucleares e órgãos de controle nuclear internacionais, bem como sobre a posição da Agência Internacional de Energia Nuclear sobre este assunto, de maneira a demonstrar que a pesquisa regulatória já faz parte da demanda nacional e deve ser fortalecida quando da criação de uma agência reguladora nuclear em nível nacional.

0 conhecimento necessário para a condução de avaliações mais complexas na área nuclear no Brasil é adquirido, em parte, em cursos de mestrado e doutorado oferecidos em poucas universidades brasileiras, onde temas não exaustivos são ensinados aos alunos, entre os quais: neutrônica (criticalidade), transporte de massa em ar, solo e água, durabilidade de barreiras de engenharia, detectores de radiação, blindagem, cálculo estrutural, transporte, 
fabricação/testes de embalagens, vibrações, cálculo de dose, métodos numéricos, analíticos e semi-analíticos (GITT- Técnica da Transformada Integral Generalizada).

Em algumas áreas da CNEN, por iniciativa de alguns servidores, inúmeros códigos/programas computacionais foram desenvolvidos e utilizados nas diversas avaliações de segurança feitas e que viabilizaram a emissão de diversos pareceres técnicos para a concessão de licenças e autorizações. Destacam-se também a importância de orientações e co-orientações de teses e dissertações de mestrado e doutorado em assuntos de interesse da CNEN voltados para a área de segurança e que, muitas vezes, resultaram nos modelos de avaliação usados pelos técnicos da CNEN/DRS para a emissão de pareceres técnicos, podendo ser citados como exemplo as seguintes teses:

- Célula quente para desmonte de medidores nucleares (REIS, 2000);

- Método multigrupo do albedo aplicado a blindagem de radiações nêutron-gama acoplados (SILVA, 2001);

- Análise das radiações nêutron gama acopladas, aplicada à blindagem com várias camadas pelo método multigrupo do albedo (DUNLEY, 2002);

- Otimização da Radioproteção (TEIXEIRA, 2003);

- Metodologia de descoberta do conhecimento em banco de dados para ambiente eletromagnético das comunicações (LONGHI, 2003);

- Modelo para avaliação dos limites de liberação de rejeitos radioativos na rede de esgoto (SHU, 2005);

- Avaliação de modelos de impacto ambiental para deposição de resíduos sólidos contendo radionuclídeos naturais em instalações mínero-industriais (P0NTEDEIR0, 2006);

- Aplicação do código computacional HYDRUS-1D na avaliação de segurança de depósitos de rejeitos radioativos - estudo de caso: repositório de rejeitos radioativos de Abadia de Goiás (FELICE, 2013);

- Modelagem Lagrangeana da dispersão atmosférica de radionuclídeos e sistemas de informação geográfica como ferramentas de suporte ao planejamento de emergência na área de influência do complexo nuclear de Angra dos Reis. (SILVA, 2013).

Ressalta-se que a própria IAEA utiliza-se de grupos de pesquisa regulatória em diversas áreas relacionadas à segurança nuclear, através da concessão de contratos de pesquisa diretos com pesquisadores de diversos países, inclusive do Brasil, chamados de "Research Agreement" 
e que se reúnem uma vez por ano em Viena, sede da IAEA, durante em média três anos, para discutir os resultados alcançados e facilitar a elaboração de suas normativas com base em uma forte pesquisa regulatória internacional.

\section{CONCEITOS REGULATÓRIOS}

Existem três principais conceitos regulatórios introduzidos na legislação brasileira: (1) Regulamento - sob a responsabilidade do Estado e controlado por uma autoridade por ele designada, constitui-se em documento normativo que visa "estabelecer regras de caráter obrigatório e que é adotado por uma autoridade” (ABNT, 1998); (2) Regulamento Técnico - sob a responsabilidade do Estado e controlado por uma autoridade por ele designada, constitui-se em documento normativo que visa "estabelecer requisitos técnicos, seja diretamente, seja pela referência ou incorporação do conteúdo de uma norma, de uma especificação técnica ou de um código de prática”; (ABNT, 1998), e (3) a Norma Brasileira, documento normativo de caráter consensual, elaborado e emitido no âmbito do Fórum Nacional de Normalização, papel exercido pela Associação Brasileira de Normas Técnicas, (ABNT, 2006) não tendo caráter obrigatório.

A conceituação de regulamento técnico foi estabelecida em 1988 pelo Conselho Nacional de Metrologia, Normalização e Qualidade Industrial (CONMETR0, 1992), que acordou a seguinte definição: "Regulamento Técnico é um documento que estabelece características de um produto ou processo a ele relacionado e métodos de produção, incluindo as cláusulas administrativas aplicáveis, com as quais a conformidade é obrigatória. Esse documento pode, também, incluir ou tratar exclusivamente de requisitos para terminologia, símbolos, embalagens, marcação, rotulagem e como se aplicam a um produto, processo ou método de produção”.

\section{A IMPORTÂNCIA DA PESQUISA REGULATÓRIA PARA A ÁREA DE SEGURANÇA DE UMA AGENCIA REGULADORA NUCLEAR}

No futuro, grande parte das empresas em todo o mundo deve continuar dirigindo seus maiores esforços na gestão estratégica de riscos para o alinhamento de suas atividades com as questões regulatórias e seu cumprimento, principalmente, em um cenário que se mostra cada vez mais globalizado. Este será, inclusive, o desafio mais significativo para as organizações, de acordo com o relatório de uma empresa de consultoria internacional (Ernest \& Young, 2010). 
Esse relatório aponta os dez maiores riscos em 2010 e as principais ameaças nos próximos cinco anos em 12 segmentos de negócios, sendo a regulamentação considerada o risco de maior impacto na indústria.

Assim, é de fundamental relevância que os órgãos reguladores governamentais brasileiros dediquem parte de seu tempo à pesquisa regulatória, inclusive realizando um estudo inicial para identificar, dentre as normas e regulamentos existentes no país, nas mais diversas áreas tecnológicas, aquelas que necessitam de revisão, levando-se em conta não somente os documentos internacionais existentes, mas, principalmente, as características de nosso país e de nossos produtos.

0 setor nuclear tem crescido no Brasil, quer seja pelas aplicações industriais, médicas e de pesquisa, como pela geração de energia em reatores nucleares de potência, envolvendo as etapas para fabricação do combustível (mineração de urânio, conversão em UF6, enriquecimento isotópico, reconversão em U02, fabricação de pastilhas e a montagem do elemento combustível nuclear) e deposição segura de rejeitos radioativos.

Nesse contexto, a pesquisa regulatória é fundamental para o desenvolvimento de ferramentas matemáticas capazes de serem aplicadas nos processos de avaliação de segurança dessas instalações, ou ainda, de depósitos de rejeitos radioativos, incluindo a seleção do local para construção desses depósitos.

Independentemente da discussão sobre a necessidade de criação de uma Agência Reguladora Nuclear, é indiscutível a importância da pesquisa regulatória para as áreas de segurança nuclear e proteção radiológica a ser fomentada pela CNEN ou pela Agência Reguladora Nuclear.

A título de ilustração, as duas avaliações de segurança realizadas para os depósitos de rejeitos de Abadia de Goiás, construídos para abrigarem os rejeitos radioativos contendo Cs-137 oriundos dos trabalhos de descontaminação da cidade de Goiânia, em face ao acidente radiológico ocorrido em setembro de 1987, foram feitas baseadas em modelos de avaliação de segurança inteiramente nacionais, desenvolvidos por servidores da CNEN na plataforma MATHEMATICA (Heilbron Filho, 1997). Em 2013, uma terceira avaliação independente dos depósitos de Goiânia foi feita utilizando-se o código HYDRUS (Felice, 2013), cujos resultados comprovaram os resultados anteriormente obtidos. 


\section{A IMPORTÂNCIA DA PESQUISA REGULATÓRIA NO ÂMBIT0 DAS AGÊNCIAS REGULAD0RAS INTERNACIONAIS}

Tamanha é a importância da pesquisa regulatória no mundo para a área de segurança, que os principais órgãos reguladores americanos - o NRC (Nuclear Regulatory Commission Comissão Reguladora Nuclear) e a EPA (Environmental Protection Agency - Agência de Proteção Ambiental) - possuem diretorias de pesquisa específicas: o "Office of Research and Development" (Escritório de Pesquisa e Desenvolvimento), no caso do EPA, e o "Office of Nuclear Regulatory Research” (Escritório de Pesquisa Regulatória Nuclear), no caso do NRC; bem como diversos centros de pesquisa e laboratórios dedicados inteiramente à pesquisa regulatória.

É evidente, portanto, que cada agência reguladora deve possuir laboratórios e diretorias de pesquisa competentes, de maneira que possam ser desenvolvidas as ferramentas matemáticas e a coleta de dados específicos, bem como que seja aprofundado o conhecimento dos aspectos fenomenológicos envolvidos nas análises e avaliações necessárias para um bom desempenho de suas funções, de modo a garantir a proteção dos trabalhadores, da população e do meio ambiente.

Ainda a título de exemplo, a diretoria de pesquisa da EPA tem como atribuição desenvolver trabalhos científicos para fornecer a base para a tomada de decisões críveis para salvaguardar a saúde humana e os ecossistemas de poluentes ambientais. 0 Escritório de Investigação e Desenvolvimento (ORD - Office of Research and Development) é o braço da pesquisa científica da EPA, cuja pesquisa de ponta ajuda a fornecer uma base sólida de ciência e tecnologia para a Agência. 0 ORD conduz seis programas de pesquisa que identificam as necessidades mais prementes de investigação em saúde ambiental. As pesquisas são desenvolvidas com a seriedade exigida por três laboratórios nacionais, quatro centros nacionais e dois escritórios localizados em quatorze instalações em todo o país.

0 cronograma da agência americana (NRC) pode ser visto em http://www.nrc.gov, o qual mostra claramente o valor que é dado pela mesma à questão da pesquisa regulatória, bem como exalta a necessidade do órgão poder multar os operadores dentro do chamado poder de polícia.

Neste sentido, cabe ressaltar que até hoje não existe na CNEN uma lista de multas aprovadas com os valores necessários.

No caso da América Latina, existem dois órgãos do setor nuclear que são independentes: 
- A Autoridade Regulatória Nuclear (ARN), na Argentina, criada em 1997, quando se desmembrou a Comissão Nuclear Argentina, através da Lei $\mathrm{N}^{0}$ 24.804. Consiste em uma entidade autárquica, subordinada à Secretária Geral da Presidência da República, modelo o qual se pretende fazer com a Comissão Nacional de Energia Nuclear brasileira;

- A Autoridade Regulatória Nacional de Proteção Radiológica, subordinada ao Ministério da Indústria, Energia e Minas, criada em 2005 através dos artigos 173 e 174 da Lei 17930, que foi desmembrada da Comissão Nacional de Energia Nuclear Uruguaia.

A tabela 1 mostra as principais agências nucleares e órgãos de controle da área nuclear no mundo, bem como os órgãos responsáveis pela pesquisa regulatória, demonstrando a importância do tema.

Um panorama sobre os demais países da América Latina que não possuem uma agência reguladora independente para o setor nuclear é mostrado a seguir:

- Na Venezuela, as atividades de regulação, pesquisa e fomento estão dentro de um único órgão, o Instituto Venezuelano de Pesquisas Científicas, localizado na cidade de Caracas;

- Na Bolívia, as atividades de regulação, pesquisa e fomento estão dentro de um único e pequeno órgão, o IBTEN (Instituto Bolivariano de Ciência e Tecnologia), situado em La Paz e subordinado ao Ministério de Ciência e Tecnologia;

- No Paraguai, essas atividades são de responsabilidade da Comissão Nacional de Energia Nuclear Paraguaia, localizada na cidade de Assunção e subordinada à Universidade de Assunção;

- No Equador, o órgão responsável é a Subsecretaria de Pesquisa e Aplicação Nuclear (SCIAN), subordinada ao Ministério de Energia;

- Na Colômbia, a organização Autoridade Regulatória Nuclear é o órgão responsável, subordinada ao Ministério das Minas e Energia (MME);

- No Chile, o órgão responsável é a Comissão Chilena de Energia Nuclear (CCHEN), situada em Santiago. Seu programa nuclear é mais desenvolvido, contando com um reator de pesquisa e quantidade de servidores adequada;

- No Peru, o Instituto Peruano de Energia Nuclear (IPEN), situado em Lima, é o órgão responsável, subordinado ao Ministério das Minas e Energia. Possui um reator de pesquisa e quantidade de servidores adequada. 
Tabela 1. Agências reguladoras nucleares internacionais.

\begin{tabular}{|c|c|c|c|c|c|c|c|c|c|c|c|c|}
\hline DEVERES & BÉLGICA & CANADÁ & FINLÂNDIA & FRANÇA & ALEMANHA & HUNGRIA & ITÁLIA & JAPÃ0 & EUA & ESPANHA & SUÉCIA & SUIÇA \\
\hline $\begin{array}{c}\text { Assessoria ao } \\
\text { Governo }\end{array}$ & FANC & CNSC & STUK & ASN & KTA, RSK e SSK & $\mathrm{AECC}$ & TCNSHP & NSC & $\begin{array}{l}\text { NRC } \\
\text { e DOE }\end{array}$ & CSN & $\begin{array}{l}\text { KASAM, SKI } \\
\text { e SSI }\end{array}$ & $\begin{array}{c}\text { HSK, KSA, } \\
\text { AGNEB e KNE }\end{array}$ \\
\hline Licenciamento & $\begin{array}{l}\text { FANC e } \\
\text { MINT }\end{array}$ & CNSC & $\begin{array}{c}\text { Governo } \\
\text { (Parlamento + } \\
\text { Municípios) }\end{array}$ & $\begin{array}{l}\text { Governo, } \\
\text { orientado } \\
\text { pela ASN }\end{array}$ & $\begin{array}{l}\text { Proprietário da } \\
\text { terra }\end{array}$ & $\begin{array}{l}\text { Parlamento; } \\
\text { SPHAMOS; } \\
\text { HAEA e } \\
\text { outras } \\
\text { autoridades } \\
\text { especiais }\end{array}$ & $\begin{array}{l}\text { MoPA e } \\
\text { APAT }\end{array}$ & $\begin{array}{l}\text { METI e } \\
\text { MEXT }\end{array}$ & $\mathrm{NRC}$ & MITYC & $\begin{array}{c}\text { Governo, } \\
\text { aconselhad } \\
\text { o pela SKI e } \\
\text { SSI }\end{array}$ & $\begin{array}{c}\text { Federal } \\
\text { Council or } \\
\text { UVEK } \\
\text { (assessorado } \\
\text { por HSK, KSA e } \\
\text { AGNEB) }\end{array}$ \\
\hline Inspeção & FANC & CNSC & STUK & ASN & $\begin{array}{c}\text { Proprietário da } \\
\text { terra e BFS }\end{array}$ & $\begin{array}{c}\text { SPHAMOS; } \\
\text { HAEA e } \\
\text { outras } \\
\text { autoridades } \\
\text { especiais }\end{array}$ & APAT & $\begin{array}{l}\text { METI e } \\
\text { MEXT }\end{array}$ & $\mathrm{NRC}$ & CSN & SKI e SSI & HSK \\
\hline Multas & FANC & CNSC & STUK & ASN & $\begin{array}{c}\text { Proprietário da } \\
\text { terra e BFS }\end{array}$ & $\begin{array}{c}\text { SPHAMOS; } \\
\text { HAEA e } \\
\text { outras } \\
\text { autoridades } \\
\text { especiais }\end{array}$ & APAT & $\begin{array}{l}\text { METI e } \\
\text { MEXT }\end{array}$ & $\mathrm{NRC}$ & CSN & SKI e SSI & HSK \\
\hline $\begin{array}{l}\text { Pesquisa } \\
\text { Regulatória }\end{array}$ & $\begin{array}{l}\text { FANC e } \\
\text { outros }\end{array}$ & $\mathrm{CNSC}$ & STUK & ASN & - & PURAM & $\begin{array}{l}\text { APAT e } \\
\text { SOGIN }\end{array}$ & NUM0 & $\mathrm{NRC}$ & $\begin{array}{c}\text { CSN e } \\
\text { ENRESA }\end{array}$ & $\begin{array}{l}\text { SKB, SKI e } \\
\text { SSI }\end{array}$ & PSI e NAGRA \\
\hline
\end{tabular}

Fonte: Heilbron (2016).

FANC - Federal Agency for Nuclear Control; MINT - Ministry of Interior; CNSC - Canadian Nuclear Safety Commission; STUK - Radiation and Nuclear Safety Authority; ASN - Autorité de Sûreté Nucléaire; KTA - Nuclear safety Standard Commission; RSK - Reactor Safety Commission; SSK - Radiation Protection Commission; BFS - Federal Office for Radiation Protection; AECC - Atomic Energy Coordination Council; SPHAMOS - State Public health and Medical Officer’s Service; HAEA - Hungary Atomic Energy Authority; PURAM - Public Agency for Radioactive Waste Management; TCNSHP - Technical Commission for Nuclear Safety and Health Protection; MoPA Ministry for Public Activities; APAT - National Agency for Environmental Protection and Technical Services; SOGIN - Society for Management Nuclear Installations; METI - Ministry Economic and Industry; MEXT - Ministry of Education, Science and Technology; NUMO - Nuclear Waste Management Organization; NRC - Nuclear Regulatory Commission; DOE - United States Department of Energy; CSN - Nuclear Safety Council; MITYC - Ministry of Industry, Tourism and Comerce; ENRESA - Spanish National Company for Radioactive Waste; KASAM - Swedish National Council for Nuclear Waste; SKI - Swedish Nuclear Power Inspectorate; SSI-Swedish Radiation Protection Institute; HSK - Swiss Federal Nuclear Safety Inspectorate; KSA - Swiss Federal Nuclear Safety Commission; AGNEB - Interdepartmental WG on Radioactive WM; KNE - Nuclear Waste Management Sub-Commission of the Federal Geological Commission; UVEK - Federal Department for Environment, Transport, Energy and Communication; PSI - Paul Scherrer Institute; NAGRA - National Cooperative for the disposal of Radioactive Waste.

Revista Internacional de Ciências, v. 07n. 01, p. 84-99, jan-jun, 2017 
A pesquisa regulatória é de grande importância para a área nuclear, independentemente do país que a utiliza, uma vez que serve, entre outros aspectos, para revalidar os modelos utilizados pelos operadores para fins de emissão de licenças e/ou autorizações pela autoridade regulatória; para adaptar modelos internacionais de avaliação de segurança às características ambientais de cada país, para fins regulatórios, como também para validar limites normativos internacionais em normas nacionais, evitando assim as chamadas barreiras não alfandegárias, isto é, quando se estabelece em uma normativa internacional um determinado limite, por exemplo, de resistência de um aço para fabricação de um embalado de rejeitos radioativos, 0 qual apenas um país fabrica, cuja justificativa técnica para o estabelecimento desse limite não é necessária do ponto de vista de segurança.

- Em alguns países mais avançados, onde existem institutos de pesquisa de grande porte na área nuclear, a autoridade regulatória nuclear às vezes subcontrata serviços técnicos de pesquisa regulatória desses institutos, evitando assim gastos desnecessários. Entretanto, deve-se ter muito cuidado com esse tipo de contratação de serviços prestados, pois, como no caso do Brasil, os institutos nucleares existentes (IPEN, CDTN e IEN) possuem reatores nucleares de pesquisa e instalações que precisam ser licenciadas/certificadas pela Diretoria de Radioproteção e Segurança Nuclear da CNEN, o que, consequentemente, caracterizaria conflito de interesses qualquer tipo de uso desses institutos para prestarem serviços de pesquisa regulatória.

\section{A IMPORTÂNCIA DA PESQUISA REGULATÓRIA NO ÓRGÃ0 REGULADOR, SEGUND0 A AGÊNCIA INTERNACIONAL DE ENERGIA ATÔMICA (IAEA)}

Outro órgão importante de nível internacional e do qual o Brasil faz parte (estado membro), e que reconhece a importância da pesquisa regulatória conduzida pelos órgãos regulatórios é a Agência Internacional de Energia Atômica (IAEA).

Esse organismo, ao redigir seus regulamentos que servem de recomendação internacional, convoca pesquisadores das autoridades regulatórias nucleares para participarem de encontros técnicos ou contratos de pesquisa para desenvolver trabalhos sobre temas relevantes à área de segurança, com o objetivo de validar suas decisões normativas. Por exemplo, a participação de pesquisadores brasileiros nesses encontros resultou no desenvolvimento de metodologias para a avaliação de segurança de depósitos finais de rejeitos radioativos (IAEA, 
2004) e no estabelecimento de limites de isenção menos restritivos para transporte de minérios com urânio e tório associados, evitando prejuízo econômico para o Brasil (IAEA, 2013).

A IAEA e as Convenções de Segurança são muito claras quando estabeleceram que, ao se elaborar uma política nacional e uma estratégia para a autoridade competente, devem ser levados em consideração:

- 0 objetivo de segurança fundamental e os princípios fundamentais de segurança estabelecidos pela IAEA (2006);

- A conexão entre os instrumentos jurídicos internacionais, como convenções e outros instrumentos internacionais pertinentes;

- A especificação do âmbito do quadro governamental, legal e regulamentar para a segurança;

- A necessidade e provisão de recursos humanos e financeiros;

- A provisão de recursos para formação de pesquisadores;

- Os mecanismos adequados para levar em conta a evolução social e econômica;

- A promoção de liderança e gestão para a segurança, incluindo a cultura de segurança.

Assim, a autoridade competente deve ter os necessários recursos humanos e financeiros para cumprir com todas as suas responsabilidades e não apenas uma parte da responsabilidade. As habilidades humanas devem abranger todas as áreas de conhecimento necessárias para garantir a segurança.

Deve ser salientado ainda que, como mostrado acima, a autoridade competente precisa promover a pesquisa regulatória, não apenas para desenvolver e melhorar as normas utilizadas no país, mas também para desenvolver seus próprios modelos de avaliação de segurança independentes.

A IAEA (2002) também afirma que, "No entanto, pode haver situações em que a pesquisa e o desenvolvimento do operador são insuficientes ou em que o organismo regulador exige pesquisa e desenvolvimento independente para confirmar os achados importantes específicos".

A entidade reguladora pode precisar realizar ou solicitar trabalho de pesquisa e desenvolvimento em apoio às suas funções de regulação em áreas como técnicas de inspeção e métodos analíticos, ou no desenvolvimento de novos regulamentos e guias. 
A estrutura organizacional do órgão regulador deve refletir essas necessidades de pesquisa e desenvolvimento, através do estabelecimento de uma unidade de pesquisa ou pelo recrutamento de pessoal, os quais possam definir as necessidades de pesquisa e desenvolvimento, bem como iniciar, coordenar e monitorar o trabalho necessário, e avaliar os resultados.

Independentemente de como a pesquisa é realizada, o órgão regulador deve garantir que ele está focado nas necessidades de regulação, seja a curto ou a longo prazo, e que os resultados sejam divulgados às unidades organizacionais adequadas”.

Importante mencionar que, para uma correta avaliação dos riscos de segurança associados com instalações nucleares, locais de deposição de rejeitos, entre outros, na maioria das vezes, torna-se necessário que os trabalhadores do órgão regulatório desenvolvam seus próprios modelos de avaliação de segurança, obviamente, independente daqueles usados por operadores em sua análise (a chamada pesquisa regulatória).

\section{A IMPORTÂNCIA DA PESQUISA REGULATÓRIA NA CNEN}

Conforme apontado na introdução, é de fundamental importância que os servidores da atual área de segurança da CNEN/DRS (Diretoria de Radioproteção e Segurança Nuclear) ou de uma futura Agência Reguladora Nuclear Brasileira, sejam capazes de desenvolver as ferramentas matemáticas adequadas, bem como identificar e determinar os dados necessários para as devidas análises, no nível e grau que a área nuclear exige em função de sua complexidade, de maneira a garantir a segurança da população brasileira e de nosso ecossistema, contra os possíveis efeitos danosos da radiação ionizante.

0 conhecimento necessário para a condução de avaliações mais complexas na área nuclear é adquirido, em parte, em cursos de mestrado e doutorado oferecidos por poucas universidades brasileiras, onde temas não exaustivos como neutrônica (criticalidade), transporte de massa em ar, solo e água, durabilidade de barreiras de engenharia, detectores de radiação, blindagem, cálculo estrutural, transporte, fabricação/testes de embalagens, vibrações, cálculo de dose, métodos numéricos, analíticos e semi-analíticos (GITT) são ensinados aos alunos. 
A CNEN, na área de gerência de rejeitos radioativos, por iniciativa de alguns servidores, possibilitou o desenvolvimento de inúmeros códigos/programas computacionais necessários às diversas avaliações de segurança feitas e que viabilizaram a emissão de diversos pareceres técnicos.

Destacam-se também a importância das orientações e co-orientações de teses e dissertações de mestrado e doutorado em assuntos de interesse da CNEN voltados para a área de segurança e que, muitas vezes, resultaram nos modelos de avaliação de segurança usados pelos técnicos da CNEN/DRS para a emissão de pareceres técnicos, podendo ser citados como exemplo as teses supracitadas no item materiais e métodos, bem como as pesquisas regulatórias, destacadas a seguir:

- Impacto radiológico no transporte de minérios;

- Análise das radiações nêutron gama acopladas, aplicada à blindagem com várias camadas pelo método multigrupo do albedo;

- Avaliação do desempenho do modelo "Califórnia Micrometeorological Model” para diagnóstico de parâmetros de camada limite atmosférica da região metropolitana do rio de janeiro;

- Contribuição ao cálculo do valor de alfa no estudo de otimização da radioproteção;

- Avaliação de impacto ambiental devido ao uso de resíduos contaminados com U e Th;

- Enriquecimento das concentrações de radônio em minas subterrâneas de fluorita e carvão do estado de Santa Catarina: critérios para avaliação dos riscos radiológicos;

- Estudo de camada limite atmosférica na região de angra dos reis através do modelo de mesoescala;

- Avaliação de segurança de instalações mínero-industriais;

- Impacto radiológico devido ao transporte de radiofármacos do IPEN-CNEN-SP;

- Estudo comparativo entre as metodologias da NCRP-49 e NCRP-147 para cálculo de blindagem de salas de raios-X;

- Estudo comparativo entre as metodologias da NCRP-49 e da NCRP-147 para cálculo de blindagem para salas de fluoroscopia;

- Metodologia para geração de cenários com fins de avaliação de segurança em repositórios próximos à superfície; 
- Caracterização de um sistema digital de aquisição de imagens radiográficas utilizando nêutrons térmicos e raios gama para inspeção de componentes mecânicos;

- Estudo sobre a inserção da zona de medição no controle legal dos instrumentos medidores de velocidade de veículos automotores;

- Fatores de escala para cálculo de inventário de rejeitos de reatores nucleares.

\section{CONCLUSÕES}

No Brasil, uma das maiores dificuldades atualmente encontradas para 0 desenvolvimento da pesquisa regulatória é a falta de reconhecimento pela própria autoridade reguladora sobre a sua importância, uma vez que confundem pesquisa básica com pesquisa regulatória, muitos inclusive, acreditando que este tipo de atividade não deveria fazer parte da autoridade regulatória. Além do mais, a falta de contratação e reposição de doutores, a falta de verba, a falta de incentivos para a publicação de artigos científicos pelo corpo técnico da área regulatória, bem como a obsolescência dos equipamentos existentes nos institutos que poderiam prestar esse tipo de serviço no caso do Brasil, contribuem enormemente para o enfraquecimento de uma área tão importante para o país e qualquer autoridade nuclear no mundo.

Um dos caminhos para o fortalecimento desta área no país seria, antes de mais nada, reconhecer essa atividade de pesquisa regulatória como um importante macroprocesso na estrutura da CNEN, em especial, na própria diretoria de radioproteção e segurança nuclear da instituição.

Órgãos com função regulatória, sendo agências reguladoras ou não, como no caso atual da CNEN, devem possuir competência para analisar a aplicabilidade de regulamentos internacionais e, até mesmo, desenvolver seus próprios modelos regulatórios, contribuindo assim para diminuir o risco relacionado à regulamentação e seu cumprimento, apontado no relatório estratégico da Ernest \& Young (2010), bem como combater as chamadas barreiras não alfandegárias.

Para tanto, é necessário que os órgãos ou agências reguladoras contenham não só uma estrutura de pesquisa e desenvolvimento adequada dentro de cada área de atuação, como também técnicos treinados em pesquisa regulatória capazes de desenvolverem os modelos necessários às requeridas avaliações de segurança. 
Para área nuclear, em particular, é importante que cursos específicos sejam criados nas universidades brasileiras ou em institutos de pesquisa e desenvolvimento do Ministério de Ciência, Tecnologia e Inovação, que incluam disciplinas de matemática avançada (solução de equações transcendentais, ajustes de curvas, inversão de matrizes, solução de sistemas lineares, não lineares, solução de equações diferenciais ordinárias, parciais, problema inverso, etc.), estatística, análise de risco, avaliação de segurança, entre outras.

Essa iniciativa contribui para que os técnicos envolvidos na área de licenciamento possam proceder às avaliações de segurança de complexidade elevada, com o nível desejado pela sociedade brasileira, garantindo assim a construção, a operação e o descomissionamento seguro de todas as instalações nucleares, radiativas, mínero-industriais e depósitos de rejeitos radioativos no país.

Como consequência, o órgão regulador poderá, com mais fundamento científico, decidir se a adoção no país dos limites e valores estabelecidos por estrangeiros em seus regulamentos técnicos é adequada ou justificável, identificando e evitando as chamadas barreiras não alfandegárias.

Igualmente importante é assegurar uma estrutura funcional para o órgão regulador nuclear que possibilite 0 adequado cumprimento de suas atribuições legais nas áreas de segurança nuclear e proteção radiológica estabelecidas na legislação brasileira.

\section{REFERÊNCIAS BIBLIOGRÁFICAS}

ABNT ISO/IEC GUIA 2. Normalização e Atividades Relacionadas - Vocabulário Geral. 1998.

ABNT ISO/IEC GUIA 2. Normalização e Atividades Relacionadas - Vocabulário Geral. 2006.

CONMETRO- RESOLUÇÃO 06/92-CONMETRO- Novo Modelo para Elaboração de Normas Técnicas no Brasil. 1992.

DUNLEY, L. S. Análise das Radiações Neutron Gama Acopladas, aplicada à Blindagem com várias Camadas pelo Método Multigrupo do Albedo. 2002. Dissertação (Mestrado em Engenharia Nuclear) - Instituto Militar de Engenharia, 2002.

ERNEST \& YOUNG Strategic Business Risk: the Top 10 Risks for Global Business in 2010- In association with the 0xford Analytica, 2010. Disponível em http://www.ey.com/GL/en/Services/Advisory/Business-Risk-Report-2010--Top-10business-risks . Acesso em 22/04/2017.

FELICE, L. B. Aplicação do Código Computacional HYDRUS-1D na Avaliação de Segurança de Depósitos de Rejeitos Radioativos -Estudo de Caso:Repositório de Rejeitos Radioativos de Abadia de Goiás- Tese de Mestrado - Instituto Militar de Engenharia - IME- Janeiro, (2013).

HEILBRON, S.R.C.L. Uma Proposta de Diretrizes Para Formulação de Estrutura Organizacional de Uma Agência Reguladora Para o Setor Nuclear Brasileiro. Dissertação (Mestrado em Sistema de Gestão), Universidade Federal Fluminense, 2016. 
HEILBRON FILHO, P. F. L.; XAVIER, A. M.; ROCHEDO, E. Safety Assessment of the Goiânia Repository - Sixth International Conference on Radioactive Waste Management and environmental Remediation-ICEM'97-Singapura-Malasia. In: Sixth International Conference on Radioactive Waste Management and environmental Remediation-ICEM'97, 1997, Singapore. - October (1997).

IAEA. Safety Assessment Methodologies for Near Surface Disposal Facilities Results of a coordinated research project Volume 1 Review and enhancement of safety assessment approaches and tools . International Atomic Energy Agency Vienna, 2004. Disponível em http://www-pub.iaea.org/MTCD/publications/PDF/ISAM/IAEA-ISAM-Voll_web.pdf. Acesso em 22/04/2017.

IAEA. Safety Standards Series- Safety Guide No. SF-1 -Fundamental Safety Principles. International Atomic Energy Agency - Vienna, 2006.

IAEA. Safety Standards Series- Safety Standards No. GS-G-1.1 -0rganization and Staffing of the Regulatory Body for Nuclear Facilities. International Atomic Energy Agency. Vienna, 2002.

IAEA. TECDOC-1728. Regulatory Control for the Safe Transport of Naturally 0ccurring Radioactive Material (NORM) - Report of a Coordinated Research Project 2007-2010. International atomic energy agency Vienna, 2013.

LONGHI, F. A. A. Metodologia de Descoberta do Conhecimento em Banco de Dados para Ambiente Eletromagnético das Comunicações. 2003. Tese (Doutorado em Engenharia de Produção) - Universidade Federal do Rio de Janeiro, 2003.

PONTEDEIR0, E. M. Avaliação de Modelos de Impacto Ambiental para Deposição de Resíduos Sólidos Contendo Radionuclídeos Naturais em Instalações Minero-Industriais. 2006. Tese (Doutorado em Engenharia Mecânica), Universidade Federal do Rio de Janeiro, 2006.

REIS, L. C. A. Célula Quente Para Desmonte de Medidores Nucleares. 2000. Dissertação (Mestrado em Engenharia Nuclear), Universidade Federal de Minas Gerais, 2000.

SHU, J. Modelo para Avaliação dos Limites de Liberação de Rejeitos Radioativos na Rede de Esgoto. 2005. Tese (Doutorado em Engenharia Nuclear). Universidade Federal do Rio de Janeiro, 2005.

SILVA, C. Modelagem Lagrangeana da Dispersão Atmosférica de Radionuclídeos e Sistemas de Informação Geográfica como Ferramentas de Suporte ao Planejamento de Emergência na Área de Influência do Complexo Nuclear de Angra dos Reis. 2013. Tese (Doutorado em Engenharia Civil), Universidade Federal do Rio de Janeiro, 2013.

SILVA, H. G. F. Método Multigrupo do Albedo Aplicado a Blindagem de radiações nêutron-gama Acoplados. 2001. Dissertação (Mestrado em Engenharia Nuclear) - Instituto Militar de Engenharia, 2001.

TEIXEIRA, P. B. Otimização da Radioproteção. 2003. Dissertação (Mestrado em Engenharia Nuclear) - Instituto Militar de Engenharia, 2003. 